\title{
In vivo efficacy of decitabine as a natural killer cell-mediated immunotherapy against isocitrate dehydrogenase mutant gliomas
}

\author{
*Xiaoran Zhang, MD, ${ }^{1}$ Wi Jin Kim, MD, ${ }^{2}$ Aparna V. Rao, PhD, ${ }^{1}$ Emade Jaman, BS, ${ }^{3}$ \\ Christopher P. Deibert, MD, ${ }^{4}$ Poorva Sandlesh, PhD, ${ }^{1}$ Katharine Krueger, BS, ${ }^{1}$ Jordan C. Allen, BS, ${ }^{1}$ \\ and Nduka M. Amankulor, MD'
}

'Department of Neurological Surgery, University of Pittsburgh Medical Center, Pittsburgh; ${ }^{3}$ University of Pittsburgh School of Medicine, Pittsburgh, Pennsylvania; ${ }^{2}$ Department of Neurological Surgery, University of California, Los Angeles, California; and ${ }^{4}$ Department of Neurological Surgery, Emory University, Atlanta, Georgia

\begin{abstract}
OBJECTIVE Isocitrate dehydrogenase (IDH) mutations are found in more than $80 \%$ of low-grade gliomas and in the majority of secondary glioblastomas. IDH mutation (IDHmut) leads to aberrant production of an oncogenic metabolite that promotes epigenetic dysregulation by inducing hypermethylation to suppress transcription of various tumor suppressor genes. Hypermethylation in IDHmut gliomas leads to transcriptional repression of NKG2D ligands, especially UL16-binding protein (ULBP)-1 and ULBP-3, and subsequent evasion of natural killer (NK) cell-mediated lysis. The demethylating agent 5-aza-2'deoxycytodine (decitabine [DAC]) is a DNA methyltransferase 1 inhibitor that prevents hypermethylation and is capable of restoring NKG2D ligand expression in IDHmut gliomas to resensitize them to NK cells. Given its capacity for sustained epigenetic reprogramming, the authors hypothesized that DCA would be an effective immunotherapeutic agent in treating IDHmut gliomas in an NK cell-dependent manner by upregulating epigenetically repressed activating NKG2D ligands in IDHmut tumors. In this study, the authors sought to use a glioma stem cell, preclinical animal model to determine the efficacy of DAC in IDHmut and IDH wild-type (IDHwt) tumors, and to characterize whether the activity of DAC in gliomas is dependent on NK cell function.
\end{abstract}

METHODS Xenograft models of IDHwt and IDHmut gliomas were established in athymic-nude mice. When tumors were grossly visible and palpable, mice were treated with either DCA or dimethylsulfoxide intraperitoneally every 7 days. Tumor sizes were measured every 2 to 3 days. After the animals were euthanized, xenografts were harvested and analyzed for the following: tumor expression of NKG2D ligands, tumor susceptibility to human and murine NK cells, immunohistochemistry for NK infiltration, and tumor-infiltrating lymphocyte characterization.

RESULTS DAC significantly inhibited the growth of IDHmut xenografts in the athymic nude mice. This effect was abrogated with NK cell depletion. Ex vivo analysis of tumor cells from harvested xenografts confirmed that DAC increased NKG2D ligand ULBP-1 and ULBP-3 expressions, and enhanced susceptibility to lysis of both human and murine IDHmut glial cells with corresponding NK cells. Immunohistochemical analysis of the xenografts indicated that DCA-treated IDHmut gliomas had a greater level of NK infiltration into the tumor compared with the negative control. Finally, DCA radically altered the tumor-infiltrating lymphocyte landscape of IDHmut glioma xenografts by increasing NK cells, dendritic cells, and M1 macrophages, while decreasing suppressive monocyte infiltration.

CONCLUSIONS DCA displayed novel immunotherapeutic functions in IDHmut gliomas. This effect was critically dependent on NK cells. Additionally, DCA significantly altered the tumor immune landscape in IDHmut gliomas from suppressive to proinflammatory.

https://thejns.org/doi/abs/10.3171/2021.11.FOCUS21489

KEYWORDS IDH mutation; glioma; glioblastoma; decitabine; immune; immunotherapy; natural killer cells

ABBREVIATIONS CFSE = carboxyfluorescein succinimidyl ester; DAC = decitabine; DMSO = dimethylsulfoxide; GSC = glioma stem cell; IACUC = Institutional Animal Care and Use Committee; IDH = isocitrate dehydrogenase; IDHmut = IDH mutation; IDHwt = IDH wild type; IgG = immunoglobulin G; NK = natural killer; PCR = polymerase chain reaction; $\mathrm{R2}-\mathrm{HG}=(\mathrm{R})-2$-hydroxyglutarate; $\mathrm{TIL}=$ tumor-infiltrating lymphocyte; ULBP = UL16-binding protein.

SUBMITTED August 17, 2021. ACCEPTED November 17, 2021.

INCLUDE WHEN CITING DOI: 10.3171/2021.11.FOCUS21489.

${ }^{*}$ X.Z. and W.J.K. contributed equally to this work. 
$\mathrm{G}$ LIOMAS are the most common malignant brain tumors and account for $80 \%$ of malignant primary brain tumors. ${ }^{1}$ Isocitrate dehydrogenase (IDH) mutations are found in more than $80 \%$ of diffuse gliomas and in the vast majority of secondary glioblastomas. ${ }^{2,3}$ Although IDH mutation (IDHmut) is generally associated with a favorable prognosis when compared with tumors with an IDH wild-type (IDHwt) genotype, IDHmut gliomas will inevitably progress to fatal, highgrade, malignant glioblastoma. ${ }^{4}$ IDHmut leads to aberrant production of (R)-2-hydroxyglutarate (R2-HG), an oncogenic metabolite that promotes epigenetic dysregulation. ${ }^{5}$ R2-HG causes hypermethylation of genomic DNA and histones, suppressing transcription of various tumor suppressor genes and altering cellular differentiation states..$^{6,7}$ This transcriptional repression through $\mathrm{R} 2-\mathrm{HG}-$ induced hypermethylation is the defining molecular feature of IDHmut gliomas. ${ }^{6}$ In addition to regulation of cell cycle and differentiation properties, epigenetic dysregulation in IDHmut gliomas also alters the expression of immune genes, thereby contributing to tumor escape from antitumor immunity. ${ }^{2,8,9}$

Natural killer (NK) cells represent the first line of antitumor immunity against neoplasia. ${ }^{10,11}$ As a critical component of the innate immune system, NK cells do not require sensitization to tumor antigens..$^{11,12} \mathrm{NK}$ cells recognize stress-induced ligands present on neoplastic cells through NK activating receptors such as NKG2D, leading to cytotoxic activity. ${ }^{13}$ We previously reported that hypermethylation of NKG2D ligands in IDHmut gliomas resulted in suppression of the cytolytic function of NK cells, allowing for tumor escape from NK-mediated immune surveillance. Specifically, hypermethylation and epigenetic repression of NK ligands in IDHmut gliomas are primarily directed at UL16-binding protein (ULBP)-1 and ULBP-3, two NKG2D ligands that belong to the ULBP class (ULBP1-ULBP6). ${ }^{9}$ Similar hypermethylation of NKG2D ligands is not present in IDHwt gliomas. These results provided the mechanistic rationale for restoration of NKG2D ligand expression as a potential therapeutic strategy for treatment of IDHmut gliomas.

Recent studies have established the use of hypomethylating agents to reset epigenetic memory in hypermethylated neoplasms. ${ }^{14}$ Therefore, we hypothesized that the demethylating agent 5-aza-2'deoxycytodine (decitabine [DAC]), a cytosine analog that induces genomic hypomethylation by inhibiting DNA methyltransferase 1, would restore expression of NKG2D ligands in IDHmut gliomas. In a previous study, we demonstrated that lowdose DAC restored expression of NKG2D ligands ULBP1 and ULBP3 in vitro and sensitized IDHmut gliomas to NK cell-mediated cytotoxicity. A recent preclinical study showed that DAC pretreatment in diffuse glioma models was effective in reducing the replicative potential of IDHmut glioma cells in vitro, and this effect was primarily ascribed to the prodifferentiation effects of DAC ${ }^{15}$ In a subsequent study, DAC was demonstrated to be effective at inducing tumor regression in vivo in an athymic mouse model of anaplastic astrocytoma, and this was thought to be secondary to increased tumor cell differentiation. ${ }^{3}$ Based on preliminary studies showing an immunomodu- latory role for DAC in IDHmut glioma cell lines, we speculated that the cytotoxic effect of DAC in vivo would be dependent on NK cell toxicity.

In this study, we used a glioma stem cell (GSC), preclinical animal model to determine the efficacy of DAC in IDHmut and IDHwt tumors. Furthermore, we set out to characterize whether the activity of DAC in gliomas is dependent on NK cell function. Our findings provide a compelling rationale and experimental support for the use of hypomethylating agents as an immune modulator in the treatment of IDHmut gliomas. In addition, the results of this study delineate an immune-related mechanism of action for DAC and its effects on the tumor microenvironment in a preclinical setting.

\section{Methods}

\section{Cells Lines and Culture}

IDH1-R132H TS603 and IDH1 wild-type TS667 are patient-derived glioma neurospheres that were generously provided to us by Dr. Timothy Chan. TS603 was derived from a patient with WHO grade III oligodendroglioma with $1 \mathrm{p} / 19 \mathrm{q}$ codeletion. TS667 was derived from a patient with WHO grade IV glioblastoma with PDGFRA amplification. These cells were propagated as GSC-like cells in NeuroCult Medium (Stemcell Technologies Inc.) according to the manufacturer's instructions. GSCs were subsequently cultured in DMEM supplemented with $10 \%$ fetal bovine serum and grown as adherent cells.

\section{DAC Treatment}

DAC (Sigma-Aldrich) was dissolved in dimethylsulfoxide (DMSO) and added to a final concentration of $1 \mu \mathrm{M}$ for 5 days. An equal volume of DMSO was used for the negative control. The medium was changed every 2 days. After 5 days, a portion of cells were harvested for experimentation. The remainder were rinsed with phosphatebuffered saline and recultured in fresh NeuroCult basal medium. Portions of cells were harvested at 3, 7, and 14 days after removing DAC or DMSO for experimentation.

\section{Real-Time Polymerase Chain Reaction}

We lysed $1 \times 10^{6}$ IDHmut and IDHwt GSCs in Trizol reagent, and RNA was isolated with chloroform extraction; ${ }^{16}$ cDNA was synthesized using an iScript cDNA synthesis kit (Bio-Rad Laboratories Inc.). Real-time polymerase chain reactions (PCRs) for ULBP-1 and ULBP-3 were performed in triplicate using Maxima SYBR Green qPCR Master Mix (Thermo Scientific) CFX96 Real-Time System Thermal Cycler (Bio-Rad Laboratories Inc.). The cycle number at which the reaction crossed an arbitrarily placed threshold $\left(\mathrm{C}_{\mathrm{T}}\right)$ was determined for each gene. The expression of each gene was quantified as a value rela-

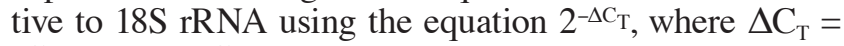
$\left(\mathrm{C}_{\mathrm{T} \text {-Target RNA }}-\mathrm{C}_{\mathrm{T}-18 \mathrm{~S} \mathrm{RNA}}\right)$.

\section{NK Cell-Mediated Cytotoxicity}

The flow cytometric carboxyfluorescein succinimidyl ester (CFSE)/7-aminoactinomycin cytotoxicity assay was performed as described. ${ }^{17}$ Glioma cells $\left(2.5 \times 10^{5}\right)$ were 
labeled with $500 \mathrm{nM}$ CFSE in phosphate-buffered saline for 15 minutes at $37^{\circ} \mathrm{C}$. CFSE-labeled target cells $(25,000$ cells) were cultured with an immortalized human NK cell line, NK-92MI (ATCC), at an effector-to-target ratio of 1:5. After 5 hours of incubation, cells were stained with $0.25 \mathrm{mg} / \mathrm{mL}$ 7-aminoactinomycin and analyzed immediately by flow cytometry.

\section{Mouse Xenograft and Treatment}

Xenograft models of IDHwt and IDHmut gliomas were established using TS667 and TS603 GSCs, respectively. IDHwt GSCs $\left(1 \times 10^{6}\right)$ were suspended in phosphate-buffered saline and combined with Matrigel matrix (Corning Inc.) at a 1:2 ratio to constitute a total volume of $100 \mu \mathrm{L}$. One hundred microliters of the glioma cell and Matrigel mixture was injected subcutaneously into the right flank of a female athymic BALB/c (Charles River) or Black-6 (The Jackson Laboratory) nude mouse. IDHmut GSCs were prepared and injected into mice in a similar fashion. Tumor growth was measured with calipers and documented daily. When tumors reached a volume of $25 \mathrm{~mm}^{3}$, mice were treated with either DMSO or $10 \mathrm{mg} / \mathrm{kg}$ DAC intraperitoneally every 7 days. Animal protocols received Institutional Animal Care and Use Committee (IACUC) approval (protocol no. 19085737).

\section{Murine NK Depletion}

Systemic NK depletion was achieved using $100 \mu \mathrm{g}$ of anti-NK1.1 immunoglobulin G (IgG) or isotype IgG (BioLegend Inc.) for controls, given intraperitoneally to mice 2 days prior to the first treatment with DAC. One day after treatment, tail venous blood samples were collected, stained for murine NKp46 cells, and analyzed on flow cytometry to ensure NK cell depletion in the mice that received anti-NK1.1 IgG. Complete depletion of peripheral NK cells was achieved prior to DAC treatment. Tail venous blood was sampled every 7 days. Mice were given $100 \mu \mathrm{g}$ of anti-NK1.1 IgG or isotype IgG again 14 days after the initial antibody injection.

\section{Xenograft Harvest and Processing}

The mice were euthanized when tumors reached a volume of $550 \mathrm{~mm}^{3}$ or the mice were determined to be suffering by IACUC standards due to either large tumor size or ulceration. Two $5 \times 5 \times 5-\mathrm{mm}$ pieces of each tumor were dissected: one piece was fixated using $4 \%$ paraformaldehyde overnight for immunohistochemical analysis, and the other was frozen at $-80^{\circ} \mathrm{C}$ for RNA extraction. The remaining tumor was mechanically dissociated to form single-cell suspension. Lympholyte-M (Cedarlane) was used according to the manufacturer's instructions to isolate tumor-infiltrating lymphocytes (TILs) from single-cell suspension.

\section{Immunohistochemical Staining}

Tissue was fixed in $4 \%$ paraformaldehyde overnight and transferred to a $30 \%$ sucrose solution for an additional 24 hours. Afterward, the tissue was embedded in OCT compound and frozen at $-80^{\circ} \mathrm{C}$ for 24 hours. Six-micrometer-thick tissue sections were prepared on glass slides and preserved at $-80^{\circ} \mathrm{C}$. At the time of immunohistochemical staining, each slide was immersed in cold acetone solution for 10 minutes for tissue preservation and blocked with $2.5 \%$ bovine serum albumin overnight. Staining was performed with $2 \mu \mathrm{g} / \mathrm{mL}$ anti-mouse NKp46 antibody (BioLegend Inc.) at $4{ }^{\circ} \mathrm{C}$ overnight, and $100 \mu \mathrm{L}$ of Alexa Fluor Goat Anti-Mouse 596 ReadyProbe reagent (Life Technologies) was placed at room temperature and was used for secondary antibody staining. Slides were rinsed with phosphate-buffered saline 8 times and stained with DAPI Fluoromount (SouthernBiotech). Tissue sections were imaged at $\times 20$ magnification using a fluorescent microscope.

\section{TIL Staining}

TILs were incubated with the following panel of antibodies according to the manufacturer's instructions: anti-mouse CD45, anti-mouse B220, anti-mouse CD11b, anti-mouse CD11c, anti-mouse F4/80, anti-mouse Ly6C, anti-mouse Ly-6G (Gr-1), and anti-mouse CD335 (NKp46) (eBioscience). Stained samples were assayed using LSRFortessa (BD). Results were analyzed using FlowJo software (BD). NK cells were identified by CD45 and CD335 (NKp46) coexpression. B cells displayed CD45 and B220 expression; dendritic cells were characterized by CD45 and CD11c; macrophages displayed CD45, $\mathrm{CD} 11 \mathrm{~b}$, and F4/80 expression; monocytes displayed CD45, CD11b, and Ly-6C; and myeloid-derived suppressor cells expressed CD45, CD11b, and Ly-6G (Gr-1). All antibodies were used at a concentration of $4 \mu \mathrm{g} / \mathrm{mL}$ (1:50 dilution).

\section{Statistical Analysis}

Data are expressed as mean and SEM, unless otherwise specified. Data collection, analysis, and representation were performed using Microsoft Excel or Prism version 7 (GraphPad Software). Statistical differences were determined using the Student $\mathrm{t}$-test and one-way ANOVA, based on the nature of data using the Prism software.

\section{Results \\ DAC Can Cause Epigenetic Changes Without Inducing Direct Cytotoxicity}

DAC functions as an epigenetic regulator through inhibition of genomic methylation. High concentrations of DAC have been shown to be directly cytotoxic. To ensure that our results are only due to DAC's epigenetic effects, we tested a large range of DAC concentrations on both IDHwt and IDHmut cell lines. We found a marked increase in cytotoxicity at $100 \mu \mathrm{M}$ DAC, but no noticeable cell death above untreated controls at lower concentrations (Fig. 1A). From these results, we decided to use $1 \mu \mathrm{M}$ DAC to conduct our future experiments to ensure that we could detect a strong epigenetic alteration without causing cytotoxicity to the cells themselves.

\section{Epigenetic Effects of DAC Are Sustained Up to 7 Days Posttreatment}

Our group has previously shown that DAC treatment can restore high expression of NKG2D ligands, especially ULBP-1 and ULBP-3, in IDHmut cell lines to increase their susceptibility to NK cell-mediated cytotoxicity. ${ }^{9}$ To 

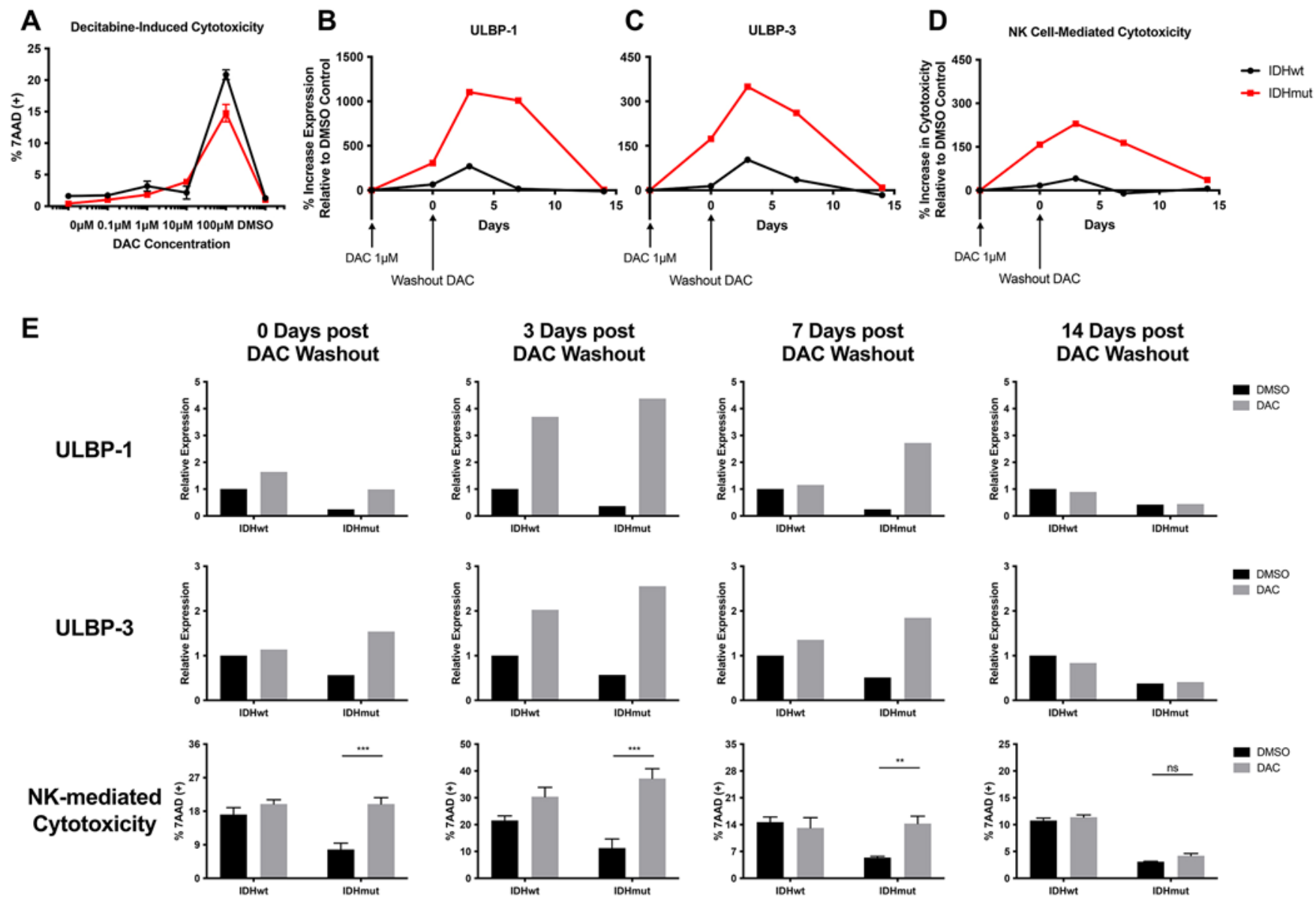

FIG. 1. DAC-induced epigenetic changes are sustained. A: Line graph showing cytoxicity of IDHwt and IDHmut GSCs at different concentrations of DAC and DMSO. B and C: Line graphs showing the percentage increases of ULBP-1 and ULBP-3 expression over the DMSO control after treatment and washout with $1 \mu \mathrm{M}$ DAC. D: Line graph showing the average percentage increase of NK-92MI-mediated cytotoxicity over the DMSO control after treatment and washout with $1 \mu \mathrm{M}$ DAC. E: Bar graphs showing ULBP-1, ULBP-3, and NK-mediated cytotoxicity after treatment and washout with $1 \mu \mathrm{M} \mathrm{DAC} .{ }^{*} p<0.05 ;{ }^{* *} p<0.01 ;{ }^{* * *} p<0.001$. 7AAD = 7-aminoactinomycin.

determine how long the epigenetic effects of DAC are sustained, both IDHwt and IDHmut cell lines were treated with $1 \mu \mathrm{M}$ DAC for 5 days. Then, DAC was washed out and the remaining cells were further cultured in fresh medium without any DAC. Cells were harvested $0,3,7$, and 14 days after washing out DAC. RNA was extracted from harvested cells and ULBP-1 and ULBP-3 expressions were determined using real-time PCR. Harvested cells were also tested for susceptibility to NK cell-mediated cytolysis.

Both ULBP-1 and ULBP-3 ligand expressions were increased with DAC treatment compared with DMSO (Fig. $1 \mathrm{~B}$ and $\mathrm{C}$ ). However, DAC induced a much more pronounced effect on IDHmut cells compared with IDHwt cells. This effect was sustained at 3 and 7 days after removing DAC but was abrogated by 14 days. Similar effects were seen with NK cell-mediated cytolysis measurements (Fig. 1D). Individual gene expression quantifications for various time points are displayed in Fig. 1E. It was interesting to note that maximum epigenetic alteration was observed 3 days after washing out DAC. These results indicate that, although DAC has a relatively short half-life in the serum, its effect at the cellular level is sustained and requires additional time to achieve the greatest transcriptional and phenotypic alteration.

\section{DAC Inhibits In Vivo Growth of IDHmut Tumors}

Given the significant epigenetic modifications on IDHmut glioma cells achieved with DAC, we decided to investigate its effects in vivo using a murine model. We established 6 IDHwt and 6 IDHmut xenograft tumors in 6-week-old female BALB/c athymic nude mice. When tumors were grossly visible and palpable, mice harboring IDHwt and IDHmut xenografts were further apportioned to receive either $10 \mathrm{mg} / \mathrm{kg}$ DAC or an equivalent volume of DMSO intraperitoneally. Given that the effects of DAC were sustained up to 7 days in our in vitro study, the animals were treated every 7 days. Tumor sizes were measured every 2 or 3 days. IDHmut tumor xenografts treated with DMSO and IDHwt tumor xenografts treated with either DAC or DMSO grew at similar rates; however, tumor growth was significantly inhibited in the IDHmut xenografts treated with DAC ( $<$ <.01) (Fig. 2A). 


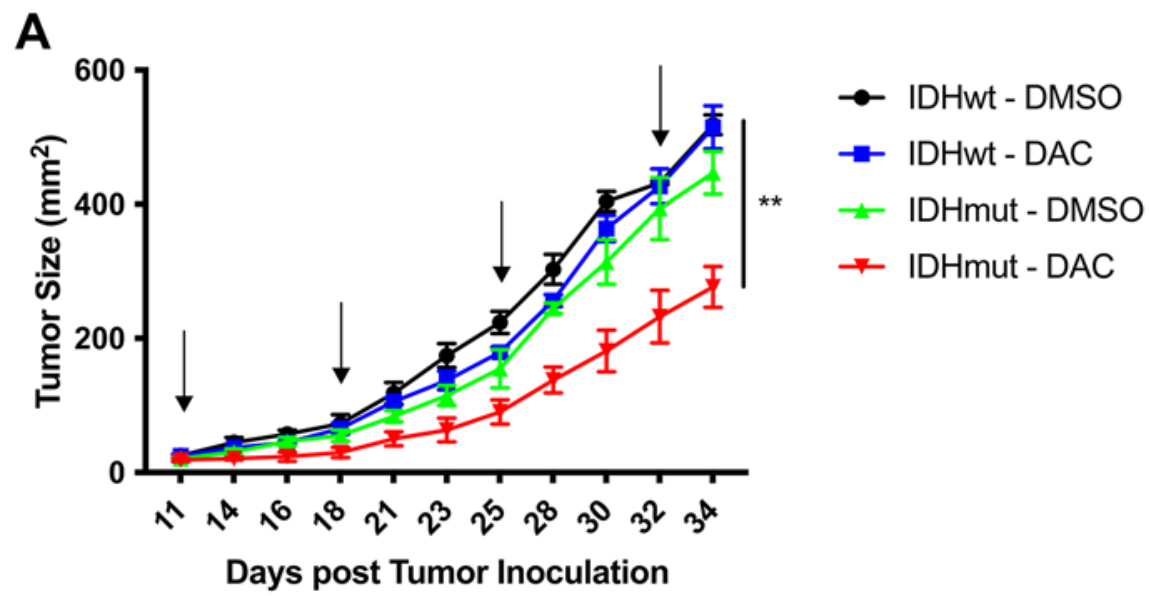

B
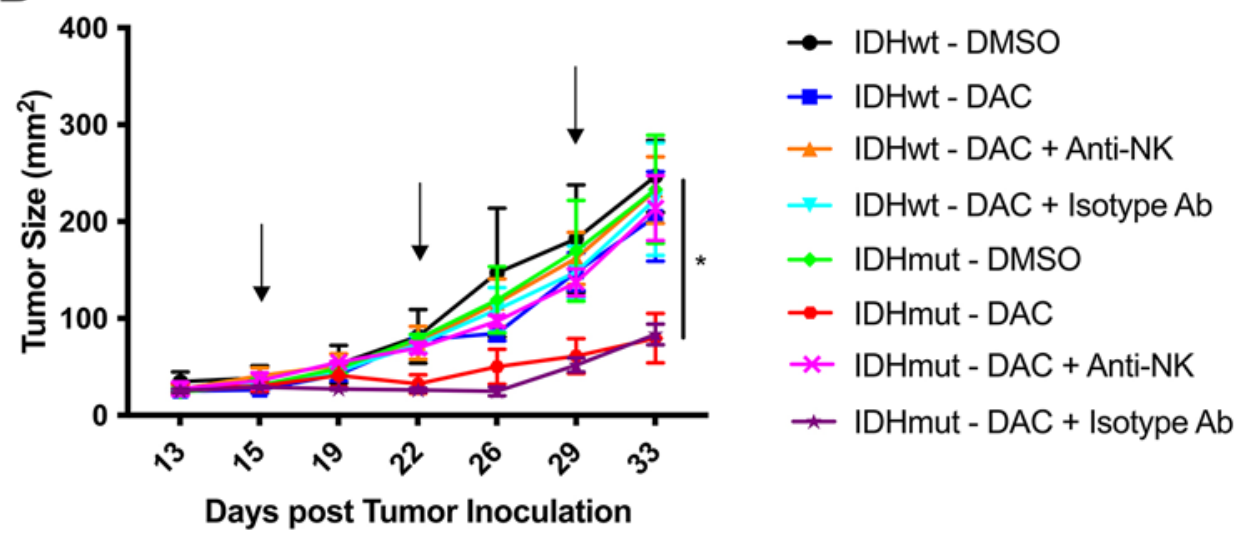

FIG. 2. DAC inhibits IDHmut tumor growth in vivo through NK cell activity. A: Line graph showing the growth curve of IDHwt and IDHmut xenograft tumors in BALB/C athymic nude mice treated with DMSO or DAC. B: Line graph showing the growth curve of IDHwt and IDHmut xenograft tumors in Black-6 athymic nude mice treated with DMSO or DAC with and without NK depletion. Arrows indicate intraperitoneal injection with either DAC or DMSO. Each curve represents an average of 3 xenograft sizes. Statistical significance was determined using one-way ANOVA. ${ }^{*} p<0.05 ;{ }^{* *} p<0.01$. Ab $=$ antibody.

\section{Inhibition of IDHmut Tumor Growth by DAC Is Mediated by NK Cells}

To elucidate the role of NK cells in DAC inhibition IDHmut tumor growth in vivo, we performed NK cell depletion in our murine xenograft model. This was done using Black- 6 athymic nude mice because anti-NK antibody and its isotype control were unavailable for BALB/c mice used in earlier experiments.

We established 12 IDHwt and 12 IDHmut xenograft tumors in 6-week-old female Black-6 athymic nude mice. Both IDHwt and IDHmut xenografts were further divided into four groups, each receiving one of the following treatment regimens: DMSO, $10 \mathrm{mg} / \mathrm{kg}$ DAC, $10 \mathrm{mg} / \mathrm{kg}$ DAC with anti-NK1.1 IgG, or $10 \mathrm{mg} / \mathrm{kg}$ DAC isotype control $\mathrm{IgG}$. When tumors were grossly visible and palpable, one group was injected with anti-NK1.1 IgG intraperitoneally, and another group was injected with isotype control IgG intraperitoneally. Two days later, each group was treated with $10 \mathrm{mg} / \mathrm{kg}$ DAC, or DMSO, every 7 days as described above. Blood samples were taken every 7 days from the mice that were given antibody injections, and their peripheral blood mononuclear cells were analyzed using flow cytometry to ensure that NK cells were depleted in those mice treated with anti-NK1.1 IgG antibody.

Similar to the results seen in our previous experiment, all IDHwt xenografts and IDHmut xenografts treated with DMSO grew at comparable rates. Interestingly, mice receiving anti-NK1.1 IgG had tumors that also grew at rates similar to those of the IDHmut xenograft treated with DMSO ( $<$ < 0.05) (Fig. 2B). This suggested that depletion of NK cells using anti-NK1.1 IgG abrogated the effects of DAC on inhibiting IDHmut tumor growth. Furthermore, IDHmut xenografts treated with isotype IgG had no discernable effect, and its tumor growth inhibition was similar to that of DAC treatment alone. Depletion of NK cells did not seem to have a significant impact on the growth of IDHwt tumors.

\section{IDHmut Xenograft Tumors Treated With DAC Have Increased NKG2D Ligand Expression}

To confirm the effect of DAC on xenograft glioma tumors, we measured NKG2D ligand expression on the tumors harvested from the above animal experiments. Real-time PCR analysis of DAC-treated IDHwt and 
A

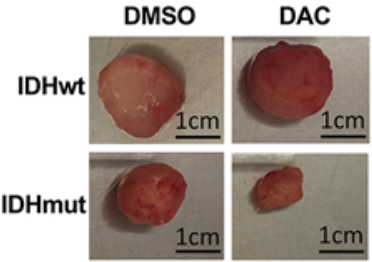

$\mathbf{F}$
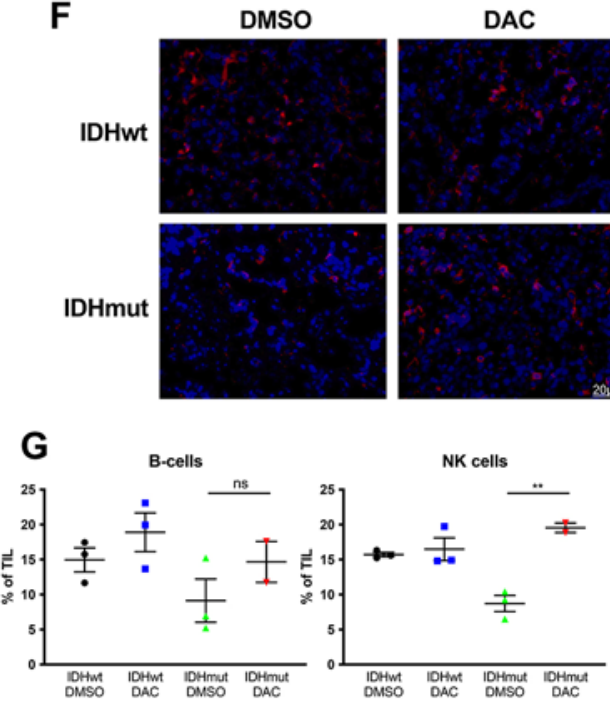

Dendritic Cells
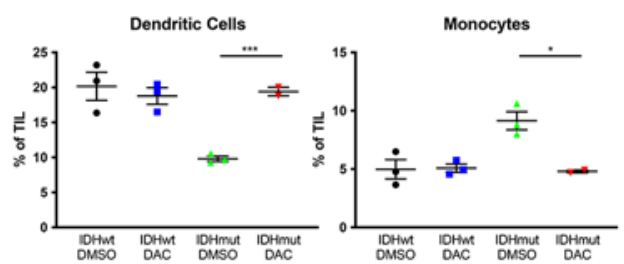

DAC

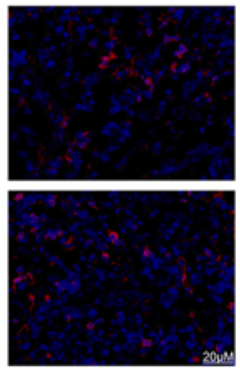

B

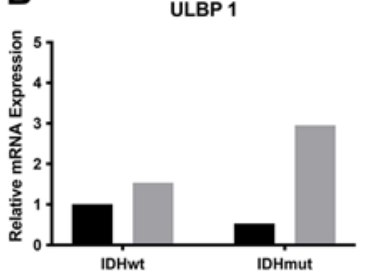

D

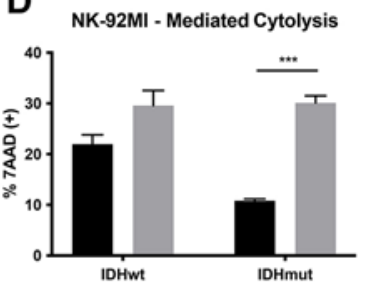

C ULBP 3

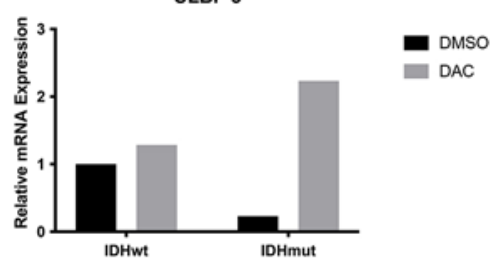

E

E Murine NK-Mediated Cytolysis

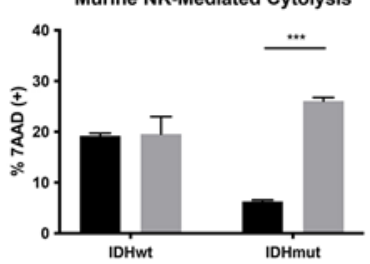

H

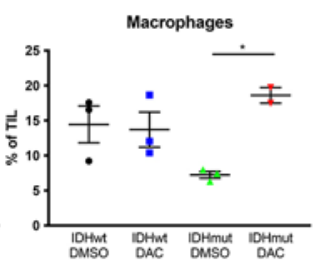

IDHwt
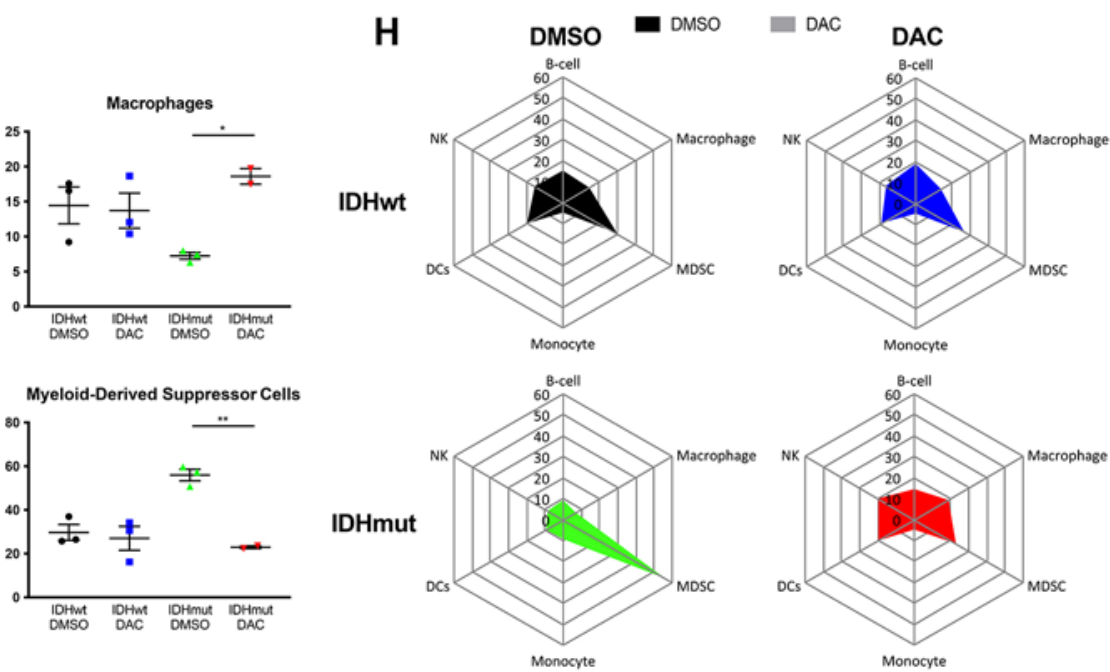

FIG. 3. DAC enhances immune susceptibility of IDHmut xenograft. A: Photographs of harvested xenograft tumors prior to processing. B and C: Bar graphs showing ex vivo ULBP-1 (B) and ULBP-3 (C) RNA expression in IDHwt and IDHmut xenograft tumors treated with DMSO or DAC. D and E: Bar graphs showing NK-92MI (D) and murine (E) NK-mediated cytotoxicity of isolated tumor cells from harvested xenografts. F: Images of immunostaining-harvested xenograft tumors depicting NK cell infiltration in harvested xenografts. Original magnification $\times 20$. Blue indicates the nuclear stain (DAPI Fluoromount) and red indicates the mouse anti-CD335 (NKp46). G and H: Boxplots (G) and radar graphs (H) showing the comparison of TIL analysis for B cells, NK cells, macrophages, dendritic cells (DCs), monocytes, and myeloid-derived suppressor cells (MDSC) from harvested xenografts. ${ }^{*} p<$ $0.05 ;{ }^{* *} p<0.01 ;{ }^{* * *} p<0.001$.

IDHmut xenografts showed that DAC increased ULBP-1 and ULBP-3 expression in both IDHwt and IDHmut cells. However, ULBP-1 and ULBP-3 expression were markedly increased in IDHmut xenografts treated with DAC compared with the increased expression seen in IDHwt xenografts (Fig. 3B and C).

\section{IDHmut Xenograft Treated With DAC Are Susceptible to NK Cell-Mediated Cytolysis Ex Vivo}

We wanted to confirm IDHwt and IDHmut xenograft susceptibility to NK cells. As expected, IDHmut xenograft cells that were treated with DAC in vivo showed increased NK-92MI-mediated cytotoxicity levels compared with the DMSO control (Fig. 3D). Similarly, IDHmut cells treated with DAC in vivo were more susceptible to murine NK cells, while IDHmut cells treated with DMSO were not (Fig. 3E). This suggested that murine NK cells were able to recognize human NKG2D ligands and destroy tumor cells in our murine model.

\section{DAC Increases NK Cell Infiltration Into IDHmut Xenografts}

Although we were convinced that DAC increased IDHmut susceptibility to NK cell-mediated cytolysis, it was uncertain if DCA could also affect NK cell chemotaxis and infiltration into the tumor. Hence, we investigated NK cell infiltration using immunohistochemistry of the harvested tumors from our murine model studies. Tissue sections of IDHwt xenografts treated with either DAC or DMSO had moderate NK cell infiltration, while sections of IDHmut xenograft that had been treated with DMSO in 
vivo had very little NK cell infiltration. Tissue sections of IDHmut xenograft that had been treated with DAC in vivo showed increased NK cell infiltration, comparable with that of IDHwt sections (Fig. 3F). These results indicated that DAC not only increases IDHmut tumor susceptibility to NK cell-mediated cytolysis, but also helps with NK cell infiltration into the tumor itself.

\section{DAC Treatment Increases TILs}

Finally, to better understand the immune landscape within the xenograft tumors and how DAC can alter it, we isolated and characterized the TILs from the harvested xenografts. Our results showed that, compared with IDHmut xenograft treated with DMSO, IDHmut xenograft treated with DAC had significantly greater infiltration with macrophages and dendritic cells in addition to NK cells (Fig. $3 \mathrm{G})$. On the other hand, DAC treatment significantly reduced monocyte and myeloid-derived suppressor cell infiltration in IDHmut xenograft compared with IDHmut xenograft treated with DMSO. There were no significant differences in the TIL landscape between IDHwt xenografts treated with DMSO or DAC. The average percentage of TIL is portrayed as radar graphs in Fig. $3 \mathrm{H}$.

\section{Discussion}

The mechanism of oncogenesis and tumor progression in IDHmut neoplasms are complex and involve metabolic derangements, alteration of cellular differentiation, perturbation of cellular DNA damage response pathways and metabolic detoxification pathways, and induction of local immune suppression in the tumor microenvironment. Notably, epigenetic modulation in IDHmut gliomas can affect transcription of immune genes and alter antitumor immunity in the process. We previously showed that the hypermethylation phenotype in IDHmut gliomas results in repressed gene expression of activating NK cell ligands of the NKG2D class, thereby dampening the cytotoxic activity of NK cells toward IDHmut glioma cells in vitro. We have also shown that, in vitro, the demethylating agent DAC can oppose the hypermethylation effect of R2-HG, resulting in increased expression of NKG2D ligands and restoration of NK cytotoxicity in IDHmut glioma cells in vitro. However, it was unclear whether DAC could induce NKG2D gene expression in vivo in IDHmut glioma models. More importantly, it was unknown whether restoration of NK cell activity induced regression of IDHmut tumors in vivo and whether the efficacy of DAC could be secondary to activation of NK cells.

DAC is a hypomethylating agent that degrades and depletes DNA methyltransferase 1 in the cell. It has been FDA approved for the treatment of myelodysplastic syndrome and has shown therapeutic efficacy in acute myeloid leukemia (AML), particularly against IDHmut AML. ${ }^{12}$ Importantly, Turcan et al. showed that pretreatment of IDHmut glioma cells with DAC prior to implantation in athymic mice resulted in significant inhibition of tumor growth. ${ }^{15}$ While this study established a global rationale for therapeutic intervention with DAC in IDHmut gliomas, the study did not establish a definitive mechanism of action for DAC. Our previous studies provided the impetus to explore a possible immune-mediated mechanism of action for DAC in IDHmut gliomas. Our current results clearly establish that, in vivo, DAC is able to dramatically suppress growth of treatment-naive IDHmut xenografts and that the efficacy of DAC is entirely dependent on the presence and activity of NK cells. Importantly, treatment of tumors in an in vivo model where cells are naive to prior pretreatment with DAC provides a physiologically relevant preclinical animal model that mirrors the temporal relationship between tumor onset and therapeutic intervention in patients. In the study by Turcan et al., ${ }^{15}$ in vitro treatment of IDHmut tumor cells prior to implantation in animals produced tumors that exhibited negligible growth following implantation. It is possible that DAC upregulated NKG2D ligand expression in vitro in that model, thereby producing immediate elimination of IDHmut tumor cells by NK cells upon implantation in xenografts. Indeed, our current study demonstrates that in vitro exposure of IDHmut glioma cells to DAC induces upregulation of NKG2D ligand expression 72 hours after treatment, suggesting that DAC-pretreated cells are primed for NK cell cytolysis upon implantation in a pretreated tumor model. In our current model, tumor growth was observed immediately upon implantation, and DAC produced a significant decrease in tumor volume in vivo but did not completely eradicate xenograft tumors. This contrasts with the DAC pretreated model by Turcan et al., ${ }^{15}$ where no significant change in tumor volume was observed after glioma cell implantation. We suspect that pretreatment of IDHmut cells with DAC prior to implantation induces upregulation of NKG2D ligands, reversal of hypermethylation, and induction of NK cell-mediated cytolysis immediately upon implantation. Interestingly, our quantitative PCR studies showed that DAC induces maximal upregulation of NKG2D ligands over 3 days and that upregulation of DAC is sustained over 3 to 7 days. This finding may have implications for DAC dosages in human trials for IDHmut glioma.

A notable finding of this study is that the efficacy of DAC in IDHmut tumors is entirely dependent on NK cell activity. Depletion of NK cells in our glioma xenograft model completely abrogates the efficacy of DAC. Thus, while DAC reverses global hypomethylation, it induces reexpression of tumor suppressor genes and modulates cellular differentiation in IDHmut malignancies. The terminal consequence of DAC exposure in the IDHmut glioma xenograft model is elimination of these cells via NK cell activity.

\section{Limitations}

There are some limitations to the current study that require additional investigation prior to human translation. First, the optimal route of administration to induce immunotherapeutic effects must be determined. A widely cited preclinical study using rabbit and rat models demonstrated that continuous intravenous infusion of DAC resulted in good CNS penetration with the CNS concentration at approximately half of the serum concentration. ${ }^{18}$ However, this finding has not been replicated in clinical studies. Furthermore, 5-azacytidine, a close analog of DAC, was found to have almost no evidence of CNS penetrance through 
intravenous administration, and sufficient CNS concentrations of 5-azacytidine were only achieved after intraventricular injection..$^{19,20}$ In future experiments, pharmacokinetic/pharmacodynamic CNS studies of DAC will need to be determined using mass spectroscopy after intravenous administration. Intraventricular administration of DAC may be a suitable option if CNS penetration is inadequate. We adopted a flank xenograft model for our current study due to several factors, including the ability to directly measure tumor size and the absence of insufficient data regarding CNS penetration of DAC. However, given the well-documented differences between CNS and peripheral tumor microenvironments, validation of our findings using an immunocompetent orthotopic murine model of a IDHmut glioma is needed.

\section{Conclusions}

Our proof-of-principle study provides evidence in the ability of DAC to modulate antitumor immune response to IDHmut gliomas through an NK cell-dependent mechanism. Further studies are needed to better understand the pharmacokinetics and bioavailability of DAC in the CNS. Validations of our findings using immunocompetent orthotopic models of IDHmut gliomas are needed to ensure the translatability of our findings. A particularly interesting future area of exploration is whether combinatorial therapy with DAC and radiation, immunotherapy, or temozolomide would lead to added therapeutic benefit.

\section{Acknowledgments}

The University of Pittsburgh holds a Physician-Scientist Institutional Award (to X.Z.) from the Burroughs Wellcome Fund.

\section{References}

1. Ostrom QT, Bauchet L, Davis FG, et al. The epidemiology of glioma in adults: a "state of the science" review. Neuro Oncol. 2014;16(7):896-913.

2. Amankulor NM, Kim Y, Arora S, et al. Mutant IDH1 regulates the tumor-associated immune system in gliomas. Genes Dev. 2017;31(8):774-786.

3. Borodovsky A, Salmasi V, Turcan S, et al. 5-Azacytidine reduces methylation, promotes differentiation and induces tumor regression in a patient-derived IDH1 mutant glioma xenograft. Oncotarget. 2013;4(10):1737-1747.

4. Yan H, Parsons DW, Jin G, et al. IDH1 and IDH2 mutations in gliomas. N Engl J Med. 2009;360(8):765-773.

5. Cairns RA, Mak TW. Oncogenic isocitrate dehydrogenase mutations: mechanisms, models, and clinical opportunities. Cancer Discov. 2013;3(7):730-741.

6. Turcan S, Rohle D, Goenka A, et al. IDH1 mutation is sufficient to establish the glioma hypermethylator phenotype. Nature. 2012;483(7390):479-483.

7. Verhaak RG, Hoadley KA, Purdom E, et al. Integrated genomic analysis identifies clinically relevant subtypes of glioblastoma characterized by abnormalities in PDGFRA, IDH1, EGFR, and NF1. Cancer Cell. 2010;17(1):98-110.

8. Kohanbash G, Carrera DA, Shrivastav S, et al. Isocitrate dehydrogenase mutations suppress STAT1 and CD8+ T cell accumulation in gliomas. J Clin Invest. 2017;127(4):1425-1437.

9. Zhang X, Rao A, Sette P, et al. IDH mutant gliomas escape natural killer cell immune surveillance by downregulation of NKG2D ligand expression. Neuro Oncol. 2016;18(10):1402-1412.
10. Herberman RB, Holden HT. Natural killer cells as antitumor effector cells. J Natl Cancer Inst. 1979;62(3):441-445.

11. Herberman RB, Nunn ME, Holden HT, Lavrin DH. Natural cytotoxic reactivity of mouse lymphoid cells against syngeneic and allogeneic tumors. II. Characterization of effector cells. Int J Cancer. 1975;16(2):230-239.

12. Kalinski P, Mailliard RB, Giermasz A, et al. Natural killerdendritic cell cross-talk in cancer immunotherapy. Expert Opin Biol Ther. 2005;5(10):1303-1315.

13. Raulet DH. Roles of the NKG2D immunoreceptor and its ligands. Nat Rev Immunol. 2003;3(10):781-790.

14. Jones PA, Issa JP, Baylin S. Targeting the cancer epigenome for therapy. Nat Rev Genet. 2016;17(10):630-641.

15. Turcan S, Fabius AW, Borodovsky A, et al. Efficient induction of differentiation and growth inhibition in IDH1 mutant glioma cells by the DNMT inhibitor decitabine. Oncotarget. 2013;4(10):1729-1736.

16. Chomczynski P, Sacchi N. Single-step method of RNA isolation by acid guanidinium thiocyanate-phenol-chloroform extraction. Anal Biochem. 1987;162(1):156-159.

17. Kim GG, Donnenberg VS, Donnenberg AD, Gooding W, Whiteside TL. A novel multiparametric flow cytometrybased cytotoxicity assay simultaneously immunophenotypes effector cells: comparisons to a 4 h 51Cr-release assay. J Immunol Methods. 2007;325(1-2):51-66.

18. Chabot GG, Rivard GE, Momparler RL. Plasma and cerebrospinal fluid pharmacokinetics of 5-Aza-2'-deoxycytidine in rabbits and dogs. Cancer Res. 1983;43(2):592-597.

19. Israili ZH, Vogler WR, Mingioli ES, Pirkle JL, Smithwick RW, Goldstein JH. The disposition and pharmacokinetics in humans of 5-azacytidine administered intravenously as a bolus or by continuous infusion. Cancer Res. 1976;36(4): 1453-1461.

20. Lester McCully C, Rodgers LT, Cruz R, et al. Plasma and cerebrospinal fluid pharmacokinetics of the DNA methyltransferase inhibitor, 5-azacytidine, alone and with inulin, in nonhuman primate models. Neurooncol Adv. 2020;2(1): vdaa005.

\section{Disclosures}

The authors report no conflict of interest concerning the materials or methods used in this study or the findings specified in this paper.

\section{Author Contributions}

Conception and design: Amankulor, Zhang, Kim, Rao, Deibert. Acquisition of data: Zhang, Kim, Rao, Sandlesh. Analysis and interpretation of data: Amankulor, Zhang, Kim, Rao, Jaman. Drafting the article: Zhang, Kim, Rao, Jaman. Critically revising the article: Amankulor, Zhang, Rao, Jaman, Sandlesh, Krueger, Allen. Reviewed submitted version of manuscript: Amankulor, Zhang, Kim. Approved the final version of the manuscript on behalf of all authors: Amankulor. Statistical analysis: Zhang, Kim, Rao, Sandlesh. Administrative/technical/material support: Amankulor, Kim, Krueger, Allen. Study supervision: Amankulor, Zhang.

\section{Supplemental Information \\ Videos}

Video Abstract. https://vimeo.com/666056811.

\section{Correspondence}

Nduka M. Amankulor: University of Pennsylvania, Philadelphia, PA.ndukaamankulor@gmail.com. 